\title{
Use of targeted therapy in patients with metastatic renal cell carcinoma: clinical and economic impact in a Canadian real-life setting
}

\author{
S. Nazha $\mathrm{MSc}^{*}$ S. Tanguay $\mathrm{MD}^{*}$ A. Kapoor $\mathrm{MD}^{\dagger}{ }^{\dagger}$ M. Jewett $\mathrm{MD}_{,}^{\ddagger}$ C. Kollmannsberger $\mathrm{MD},{ }^{\S} \mathrm{L}$. Wood $\mathrm{MD},{ }^{\prime \prime}$ \\ G. Bjarnason MD, ${ }^{\#}$ D. Heng MD, ${ }^{* *}$ D. Soulières $\mathrm{MD}_{,}^{+\dagger}$ N. Reaume $M D_{,}^{\neq \neq}$N. Basappa $M D_{,}^{\S \S}$ \\ E. Lévesque $M D,|l|$ and A. Dragomir PhD*
}

\begin{abstract}
Introduction Outside of randomized controlled clinical trials, the understanding of the effectiveness and costs associated with targeted therapies for metastatic renal cell carcinoma (mRCc) is limited in Canada. The purpose of the present study was to use real-world prospective data to assess the effectiveness and cost of targeted therapies for patients with mRCC.

Methods The Canadian Kidney Cancer Information System, a pan-Canadian database, was used to identify prospectively collected data relating to patients with mRCc. First- and subsequent-line time to treatment termination (ТтT) was determined from therapy initiation time (sunitinib or pazopanib) to discontinuation of therapy. KaplanMeier survival curves were used to estimate the unadjusted and adjusted overall survival (os) by treatment. Unit treatment cost was used to estimate the cost by line of treatment and the total cost of therapy for the management of patients with mrcc.
\end{abstract}

Results The study included 475 patients receiving sunitinib or pazopanib in the first-line setting. Patients were treated mostly with sunitinib (81\%); $19 \%$ of patients were treated with pazopanib. The median тTт in the first line was 7.7 months for patients receiving sunitinib and 4.6 months for those receiving pazopanib $(p<0.001)$. The adjusted os was 32 months with sunitinib and 21 months with pazopanib (hazard ratio: 1.61; $p<0.01$ ). The total median cost of first- and second-line treatments was $\$ 56,476$ (interquartile range: $\$ 23,738-\$ 130,447$ ) for patients in the sunitinib group and $\$ 46,251$ (interquartile range: $\$ 28,167-\$ 91,394$ ) for those in the pazopanib group.

Conclusions For the two therapies, os differed significantly, with a higher median os being observed in the sunitinib group. The cost of treatment was higher in the sunitinib group, which is to be expected with longer survival.

Key Words Renal cell carcinoma, targeted therapy, effectiveness, safety, real-world data

Curr Oncol. 2018 Dec;25(6):e576-e584 www.current-oncology.com

\section{INTRODUCTION}

In 2016, approximately 6200 Canadians were diagnosed with kidney cancer. Renal cell carcinoma (RCC) accounted for $90 \%$ of those patients, and clear cell histology accounted for $70 \%$ of $\operatorname{RCCs}^{1,2}$. Although surgery remains the optimal treatment option for localized RCC, $20 \%-30 \%$ of patients will present with metastasis at the time of diagnosis. In addition, more than $30 \%$ of patients will develop metastatic disease at some point ${ }^{3}$. The prognosis for patients with metastatic disease is poor: the estimated overall 5-year survival probability rate is less than $10 \%{ }^{4,5}$. Ultimately, 
many patients with RCC will develop metastatic disease and, based on current guideline recommendations, will require treatment with targeted therapies ${ }^{6-9}$.

Based on phase in randomized controlled trials (RCTs) and a head-to-head trial, sunitinib and pazopanib have both been approved as first-line treatments for the treatment of metastatic RCc (mRcc). The head-to-head trial showed a median on-treatment period of 7.6 months for the sunitinib group and 8.1 months for the pazopanib group, and a median overall survival (os) of 29.1 months with sunitinib and 28.3 months with pazopanib ${ }^{10}$. In 2016, new molecular discoveries (PD-1/PD-L1) led to the approval of nivolumab, reintroducing immunotherapy into the treatment algorithm for mRcc ${ }^{11}$. Treatments based on those discoveries have been shown to have activity in the first-line setting in patients with mRcc; however, sunitinib and pazopanib monotherapy will still be the standard of care for many Canadian patients $s^{6,12,13}$. Understanding the costs associated with those high-cost therapies is therefore essential, and outside of randomized controlled clinical studies, an understanding of the effectiveness and costs associated with targeted therapies for mRcc is limited in Canada. The need for data from real-life patients exposed to the contemporary targeted therapies is therefore growing. The purpose of the present study was to assess the effectiveness and cost of targeted therapies for patients with clear cell mRcc treated with sunitinib or pazopanib in the first-line setting.

\section{METHODS}

Data from the Canadian Kidney Cancer Information System (cксis) for eligible patients with mRcc who received targeted therapy were used for the analysis. The cкcis is a multicentre collaboration of 14 academic hospitals in 6 Canadian provinces. Patient characteristics collected from the cKcis were age, sex, date of Rcc and mRcc diagnoses, comorbidities, and the location and number of metastases. Treatment characteristics included start date of each treatment, type of systemic therapy, dose adjustment, and whether the patient had public or private funding. The database also provided information about the surgery type (nephrectomy or metastasectomy) and timing. Clinical, demographic, imaging, and pathology data were obtained from patient medical records at each site and were collected up to December 2016.

\section{Study Cohort}

Our study cohort consisted of patients diagnosed with clear cell mRcc after 1 January 2011, with prospectively collected data. Patients with a confirmed histologic diagnosis of mRcc, clear cell subtype, and receiving firstline targeted therapy with sunitinib or pazopanib were included. The index date was defined as the date of first prescription of either sunitinib or pazopanib (Figure 1). Characteristics of the patients were collected from the date of mrcc diagnosis until the end of the follow-up period. The analysis period spanned the index date to the end of follow-up, which was the earliest of death date, patient's last visit, or the end of the study period (31 December 2016).

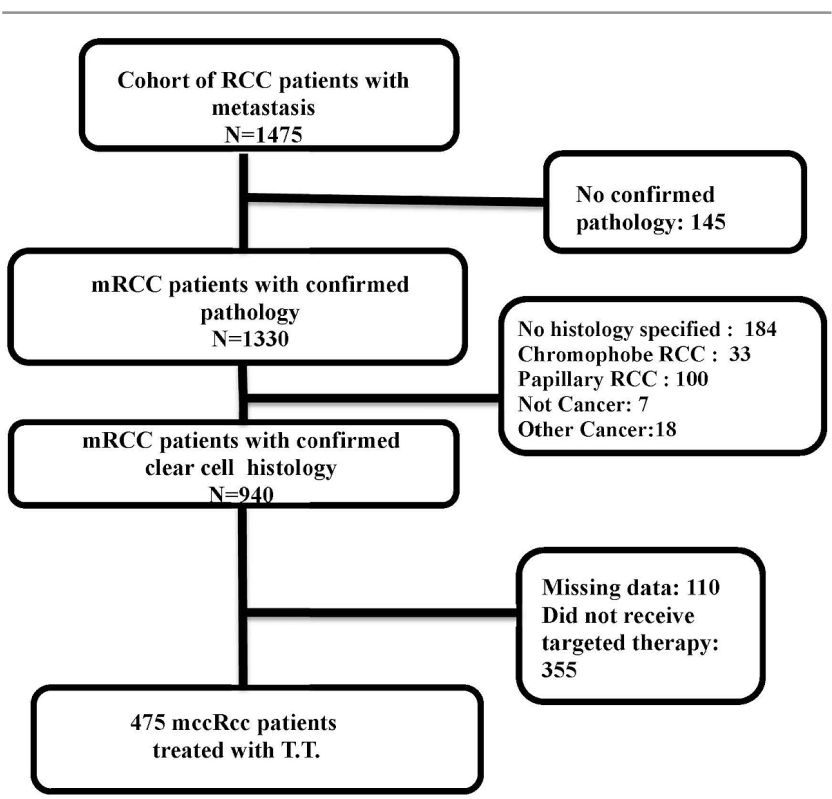

FIGURE 1 CONSORT diagram for the patient cohort. RCC = renal cell carcinoma; $\mathrm{mRCC}=$ metastatic $\mathrm{RCC} ;$ mccRcc $=$ metastatic clear cell RCC.

\section{Statistical Analysis}

Descriptive statistics are used to summarize baseline patient characteristics. Means, 95\% confidence intervals (CIS), medians, and interquartile ranges (IQRS) are used to describe continuous variables; percentages are used to describe categorical variables. The $t$-test and chi-square test were used to assess differences between the sunitinib and pazopanib groups in terms of patient demographics and disease and treatment characteristics. First- and subsequent-line time to treatment termination (TTT) was determined starting at initiation of the respective line of therapy until discontinuation of that line. The median durations of the first and second lines of treatment were derived from that analysis. Overall survival was determined from the index date until the end of follow-up.

\section{Effectiveness Estimation}

Mean and median os from the initiation of first-line targeted therapy was calculated using the Kaplan-Meier method. A Cox proportional hazards model was used to examine the effect of targeted therapy, controlling for demographics and disease and treatment characteristics. Several covariates evaluated at the time of mRCC diagnosis were considered to be potential predictors of progression: age (<65 vs. $\geq 65$ years of age), sex, Eastern Cooperative Oncology Group performance status (ECOG PS) at index date (0 or 1 vs. $>1$ ), metastasis location (lung, bones, liver, brain, lymph nodes), number of different metastasis locations (1 vs. >1), synchronous metastasis, and timing of targeted treatment initiation after the mRcc diagnosis ( $<1$ year vs. $\geq 1$ year). Surgeries such as nephrectomy before targeted treatment and metastasectomy (curative or palliative) were also included in the Cox model and were adjusted as time-dependent variables given that the surgery could have been conducted before or after initiation of the targeted treatment. In 
addition, the direct adjusted survival function was used to estimate the survival curves - and the adjusted median and mean survival—of the average patient in each group ${ }^{14}$. In that method, the direct adjusted survival function is estimated by averaging the predicted survival function for each combination of covariates. Given the high differential ratio of patients receiving sunitinib compared with those receiving pazopanib (5:1), propensity score matching was considered suboptimal and was therefore not our preferred or selected method.

The survival prognosis of patients having survived 1 year after initiation of first-line targeted therapy was estimated using 1-year conditional survival analysis. A Cox regression model was used to examine the effect of variables on os at 1 year after treatment initiation. Similarly, the direct adjusted conditional survival function was plotted.

\section{Cost Estimation}

The unit cost of the therapies was derived from the list of medications published by the Régie de l'assurance maladie du Québec. The cost of each individual line of treatment was estimated by multiplying the time on treatment for each therapy in each line by the unit cost. The total cost was estimated by summing the cost of first- and subsequent-line treatments, weighted depending on the medication used in first-line (sunitinib or pazopanib) and subsequent-line (axitinib, sunitinib, pazopanib, everolimus) therapy.

All analyses were performed using the SAS software application (version 9: SAS Institute, Cary, NC, U.S.A.). All tests were two-sided, with a significance threshold of $5 \%$.

\section{RESULTS}

\section{Study Population}

At December 2016, 1475 patients with metastatic disease diagnosed after January 2011 were identified in the сксіs database; 940 of them had confirmed clear cell histology. In the cohort of 940 patients, 38\% $(n=355)$ did not receive targeted treatment during the course of their disease, and 110 patients were excluded from the analysis because of missing key data. The final study cohort therefore consisted of 475 patients with clear cell mRcc who received either sunitinib or pazopanib as a first-line targeted treatment (Figure 1).

\section{Patient Characteristics}

Table I presents the baseline characteristics of the study cohort. Median age was 63 years, and $76.2 \%$ of the patients were men. Patients with a synchronous diagnosis of metastasis and RCC constituted $52.8 \%$ of the cohort. Median time from diagnosis of metastasis to treatment with targeted therapy was 3.4 months (IQR: 1.93-7 months), and patients were followed for a maximum of 69 months in the sunitinib group and 56 months in the pazopanib group. Mean follow-up was 25.6 months in the sunitinib group (95\% CI: 23.8 months to 27.3 months; median: 21 months; IQR: 11-39 months) and 20.4 months in the pazopanib group (95\% CI: 17.4 months to 23.5 months; median: 17 months; IQR: 10-30.5 months). Cytoreductive nephrectomy was performed in $76.9 \%$ of the patients, and $19.2 \%$ underwent metastasectomy (either palliative or curative intent) during the course of their treatment. The most common site of metastasis was lung (53.3\%), followed by bones $(19.5 \%)$,

TABLE I Characteristics of patients with metastatic renal cell carcinoma (mRCC) undergoing first-line therapy

\begin{tabular}{|c|c|c|c|c|}
\hline Characteristic & Overall & Sunitinib & Pazopanib & $p$ Value $^{\mathrm{a}}$ \\
\hline Patients (n) & 475 & 395 & 80 & \\
\hline Sex (\% men) & 76.2 & 76.9 & 72.5 & 0.39 \\
\hline Age (years) & & & & 0.09 \\
\hline Median & 63 & 63 & 65 & \\
\hline IQR & $56-70$ & $56-70$ & $57-75$ & \\
\hline $\begin{array}{l}\text { RCC Dx to metastasis }{ }^{b} \\
\text { (months) }\end{array}$ & & & & 0.21 \\
\hline Median & 2.1 & 1.5 & 6.1 & \\
\hline IQR & $0-17.5$ & $0-15.4$ & $0-29.9$ & \\
\hline $\begin{array}{l}\text { Metastasis to } 1 \mathrm{st}-\text {-line } \\
\text { treatment (months) }\end{array}$ & & & & 0.05 \\
\hline Median & 3.4 & 3.3 & 4.6 & \\
\hline IQR & $1.9-7$ & $1.9-6.3$ & $2.1-11.2$ & \\
\hline Synchronous mRCC (\%) & 52.8 & 54.7 & 43.8 & 0.08 \\
\hline $\begin{array}{l}\text { Nephrectomy before } \\
\text { targeted therapy }(\%)\end{array}$ & 76.9 & 76.7 & 85.0 & 0.13 \\
\hline Metastasectomy (\%) & 19.2 & 20.0 & 17.5 & 0.60 \\
\hline \multicolumn{5}{|l|}{ Site of metastasis ${ }^{\mathrm{c}}(\%)$} \\
\hline Lung & 53.3 & 54.5 & 47.4 & 0.26 \\
\hline Adrenal glands & 11.4 & 11.1 & 12.8 & 0.67 \\
\hline Bone & 19.5 & 19.8 & 18 & 0.70 \\
\hline Liver & 7.9 & 8.7 & 3.9 & 0.06 \\
\hline Lymph nodes & 22.5 & 21.3 & 28.8 & 0.22 \\
\hline Brain & 4.3 & 4.6 & 2.6 & 0.32 \\
\hline ECOG PS (\%) & & & & 0.02 \\
\hline 0 & 37.6 & 39.2 & 29.1 & \\
\hline 1 & 48.2 & 48.1 & 48.1 & \\
\hline$\geq 2$ & 16.1 & 12.7 & 22.8 & \\
\hline \multicolumn{5}{|l|}{$\begin{array}{l}\text { Organs with } \\
\text { metastases }{ }^{\mathrm{c}}(\%)\end{array}$} \\
\hline 1 & 68.1 & 67.9 & 69.2 & 0.72 \\
\hline 2 & 22.7 & 23.4 & 19.2 & \\
\hline$\geq 3$ & 9.2 & 8.7 & 11.5 & \\
\hline \multicolumn{5}{|l|}{ Year of mRCC Dx (\%) } \\
\hline 2011 & 7.8 & 9.4 & 0 & $<0.0001$ \\
\hline 2012 & 20.6 & 23.8 & 5.0 & \\
\hline 2013 & 20.2 & 21.0 & 16.3 & \\
\hline 2014 & 21.9 & 20.8 & 27.5 & \\
\hline 2015 & 18.7 & 15.9 & 32.5 & \\
\hline 2016 & 10.7 & 9.1 & 18.8 & \\
\hline
\end{tabular}

a Significant values shown in boldface type.

b Defined as less than 3 months from the initial diagnosis of RCC to the diagnosis of metastasis.

c Patients could have lesions at more than one site.

$\mathrm{IQR}=$ interquartile ratio; $\mathrm{Dx}=$ diagnosis; $\mathrm{ECOG} \mathrm{PS}=$ Eastern Cooperative Oncology Group performance status 
lymph nodes (22.5\%), adrenal glands (11.4\%), liver (7.9\%), and brain (4.3\%). Most patients had 1 site of metastasis (68.1\%). Most patients (85.8\%) had an ECOG PS of 0 or 1 (Table I).

Most patients (81\%) were treated with sunitinib in the first line; the remaining $19 \%$ were treated with pazopanib. The patient population in both groups was similar, except that the time from diagnosis of mRCc to treatment initiation was longer in the pazopanib group than in the sunitinib group [4.6 months (IQR: 2.1-11.2 months) vs. 3.3 months (IQR: 1.9-6.3 months), $p=0.05$ ] and that more patients in the sunitinib arm than in the pazopanib arm had an ECOG Ps of 0 or 1 ( $87.3 \%$ vs. $77.3 \%, p=0.02)$. Median age was also different in the groups (63 years for sunitinib vs. 65 years for pazopanib), but the difference was not statistically significant ( $p=0.09$, Table I).

\section{Drug Therapies}

The median ттт in the first line was 7.7 months for patients receiving sunitinib and 4.6 months for those receiving pazopanib $(p<0.001)$. Of the patients who received subsequent-line treatment $(n=191), 42.4 \%(n=81)$ received everolimus, $18.3 \%$ ( $n=35$ ) received pazopanib, $15.2 \%(n=29)$ received sunitinib, $16.2 \%(n=31)$ received axitinib, and $7.9 \%$ received other targeted therapies. The median treatment duration in the second line was 8.04, $3.25,3.32$, and 4.14 months in the sunitinib, pazopanib, everolimus, and axitinib groups respectively $(p<0.001)$. The distribution of subsequent treatment lines was similar in the first-line sunitinib and pazopanib groups. Use of everolimus was found in $19.4 \%$ and $14.4 \%$ of the first-line sunitinib and pazopanib groups respectively, and use of axitinib was found in $6.8 \%$ and $6.7 \%$ of those groups. In both groups, the proportion of patients who received no subsequent therapy was in the range of $58 \%-60 \%$ (Table II).

\section{Survival}

Median os was 30 months (IQR: 25-36 months) for patients treated with sunitinib and 19 months (IQR: 15-24 months) for those treated with pazopanib $[p=0.03$, Figure 2(A)]. After 1 year, $73.8 \%$ of patients in the sunitinib group and $72.7 \%$ of those in the pazopanib group were still alive. The corresponding direct adjusted median survival durations were, respectively, 32 months [IQR: 13 months to not reached (NR)] and 21 months [IQR: 9-53 months; Figure 2(B)]. Cox proportional hazards regression revealed that, compared with patients treated with sunitinib, those treated with pazopanib experienced a greater risk of death (HR: 1.61; 95\% CI: 1.10 to 2.36), with adjustment for potential confounding variables (Table III). Several other variables were associated with increased risk of death: ECOG PS of 2 or greater (HR: 2.05; 95\% CI: 1.43 to 2.96), brain metastasis (HR: $2.11 ; 95 \%$ CI: 1.07 to 4.14 ), and synchronous metastasis (HR: $1.50 ; 95 \%$ CI: 1.10 to 2.01). However, a time to treatment initiation exceeding 1 year from diagnosis of mRCC was associated with a decreased risk of death (HR: $0.45 ; 95 \%$ cr: 0.26 to 0.78 ). The proportional hazards assumption was assessed and validated before the multivariate regression analysis was performed.

The median 1-year conditional survival was 41 months (95\% CI: 28 months to NR) in the sunitinib group and 12

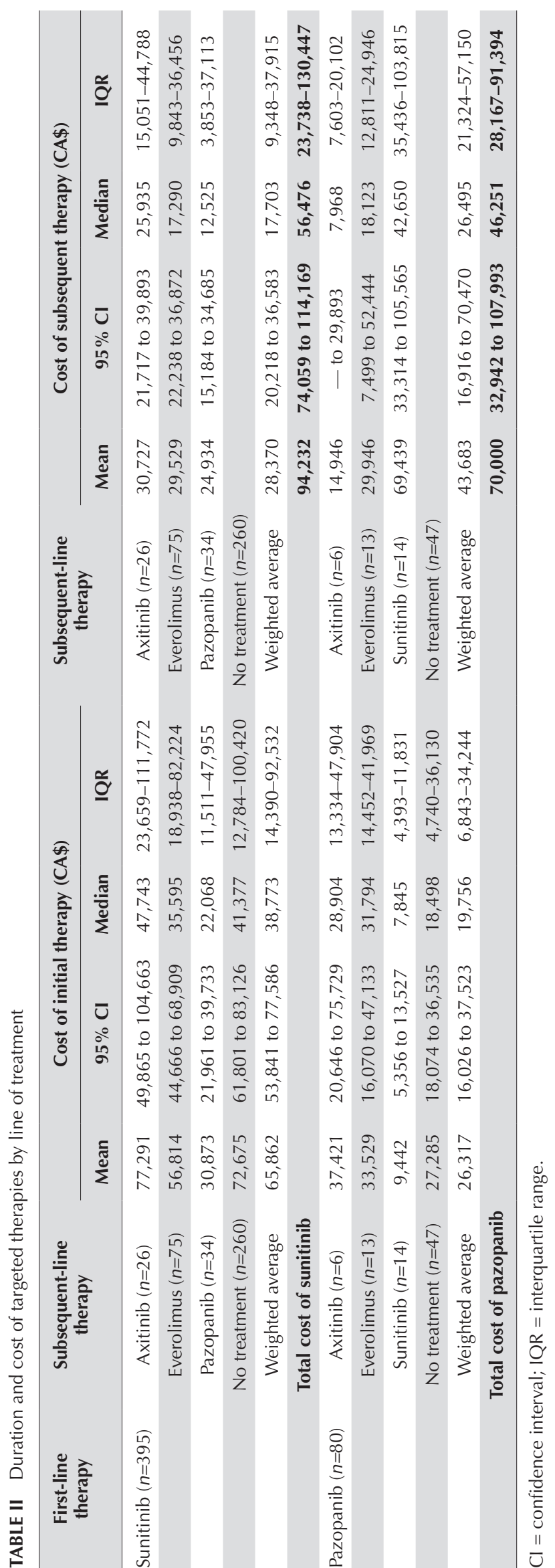


months (95\% CI: 7 months to NR) in the pazopanib group [Figure 2(C)]. Similar results were obtained for the median adjusted conditional survival: 41 months (95\% CI: 13 months to NR) with sunitinib and 13 months (95\% CI: 5 months to 30 months) with pazopanib [Figure 2(D)]. When adjusted for covariates, including those not balanced between the two arms (ECOG Ps and time to treatment initiation), patients in the pazopanib group still alive at 1 year had a higher risk of death than did their counterparts in the sunitinib group (HR: $2.48 ; 95 \%$ CI: 1.41 to 4.36 ). After 1 year, no other variables were associated with risk of death.

\section{Costs}

As shown in Table II, the median cost for treatment with 1 line of targeted therapy was $\$ 38,773$ (95\% CI: $\$ 14,390$ to $\$ 92,532$ ) for sunitinib and $\$ 19,756$ (95\% CI: $\$ 6,843$ to $\$ 34,244$ ) for pazopanib. When up to 2 lines of targeted therapy were included, the total cost was $\$ 56,476$ (95\% cr: $\$ 23,738$ to $\$ 130,447$ ) for patients treated with sunitinib in the first line and $\$ 46,251$ (95\% cr: $\$ 28,167$ to $\$ 91,394$ ) for patients treated with pazopanib in the first line. When the cost of treatment was adjusted for patient survival, the cost per month of treatment became lower for patients initiated

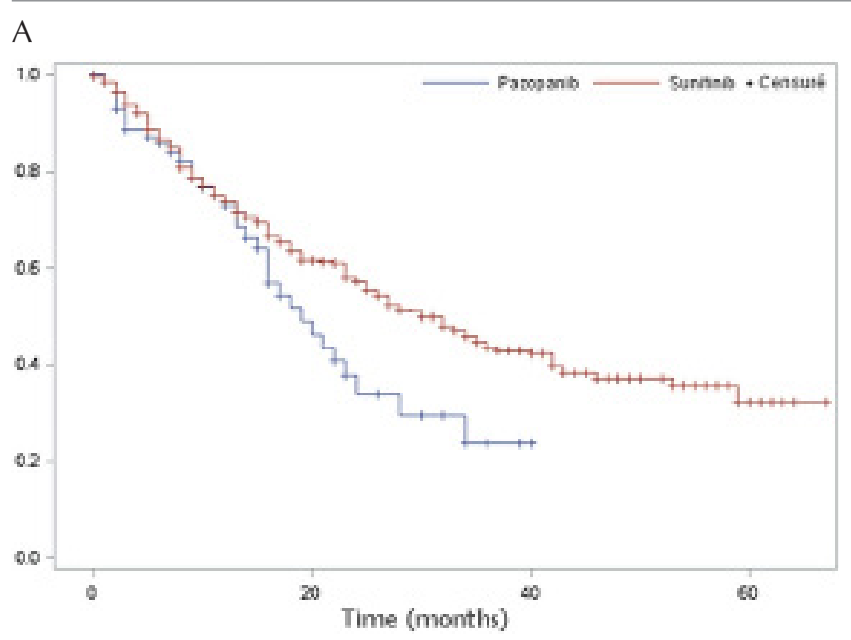

B

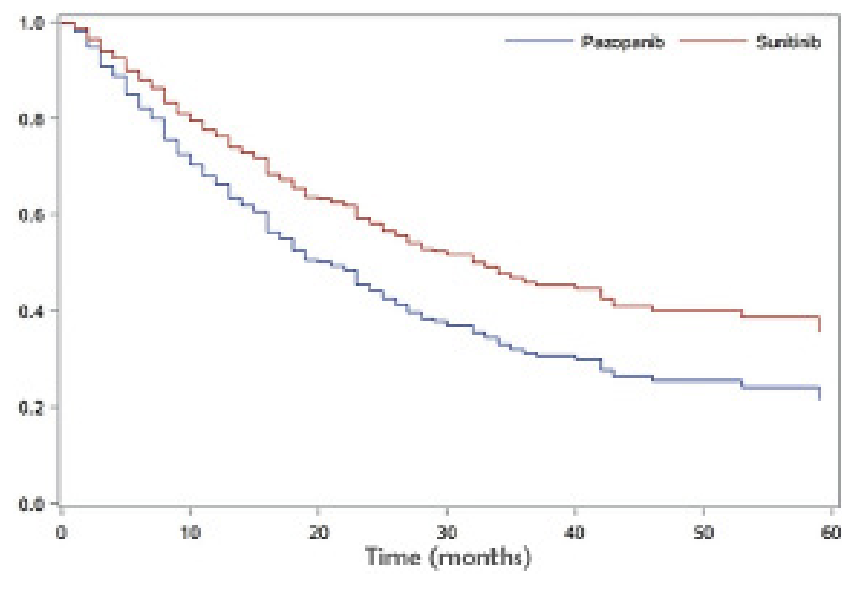

D

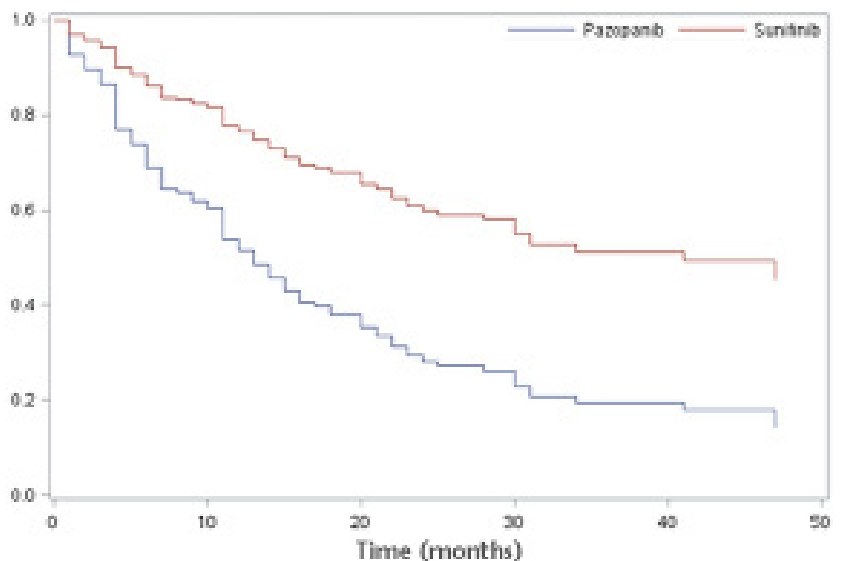

(1)

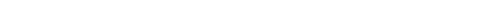

C

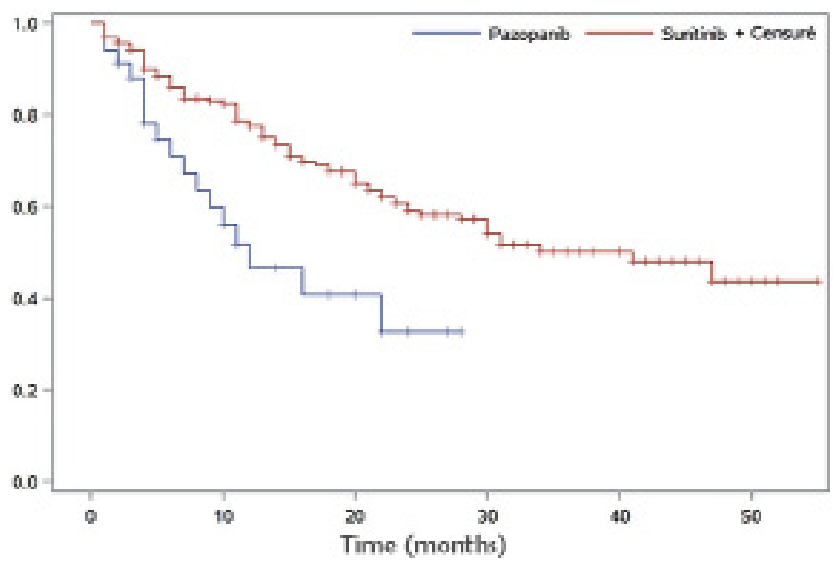

\begin{tabular}{|c|c|c|c|c|c|c|}
\hline \multicolumn{2}{|r|}{ Panel } & \multicolumn{4}{|c|}{ Overall survival } & \multirow{3}{*}{$\underset{\text { Value }}{p}$} \\
\hline & & \multicolumn{2}{|c|}{ With sunitinib } & \multicolumn{2}{|c|}{ With pazopanib } & \\
\hline & & Median & $95 \% \mathrm{Cl}$ & Median & $95 \% \mathrm{Cl}$ & \\
\hline A & Kaplan to Meier curves & 30 & 25 to 36 & 19 & 15 to 24 & 0.03 \\
\hline B & Adjusted curves & 32 & 13 to NR & 21 & 9 to 53 & 0.01 \\
\hline $\mathrm{C}$ & Conditional survival curves & 41 & $28-N R$ & 12 & 7 to $N R$ & 0.02 \\
\hline $\mathrm{D}$ & Adjusted conditional survival curves & 41 & $13-N R$ & 13 & 5 to 30 & 0.01 \\
\hline
\end{tabular}

$\mathrm{Cl}=$ confidence interval; $\mathrm{NR}=$ not reached.

FIGURE 2 Survival curves. (A) Kaplan-Meier overall survival (OS). (B) Adjusted OS. (C) Conditional OS. (D) Adjusted conditional OS. 
EFFECTIVENESS OF SUNITINIB COMPARED WITH PAZOPANIB, Nazha et al.

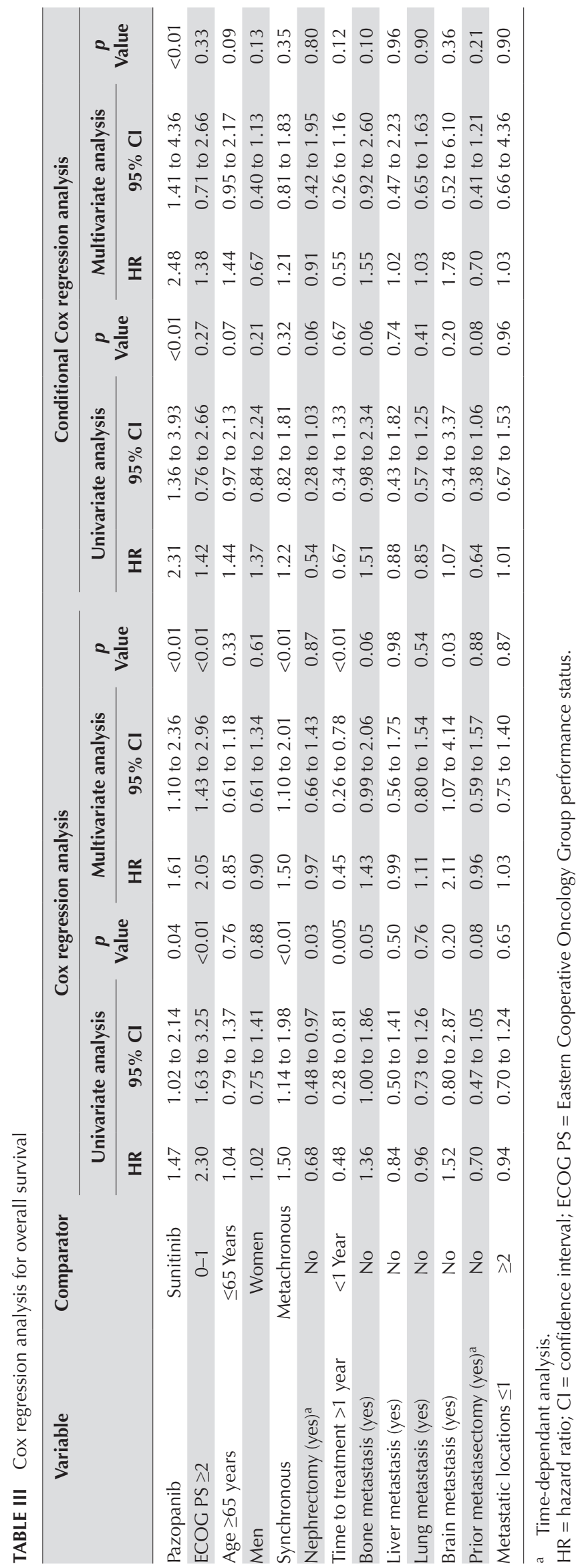

on sunitinib than for those initiated on pazopanib, because patients receiving sunitinib tended to live longer $(\$ 1,765 /$ month vs. $\$ 2,202 /$ month respectively).

\section{DISCUSSION}

Many guidelines recommend the use of targeted therapies in the first-line setting for patients with mRCC of clear cell histology ${ }^{6-9}$. However, information about the effectiveness and cost of targeted therapies for mRCc patients in real life is limited. Our ckcis database is unique in providing an in-depth look at contemporary mrcc management in academic hospitals across Canada. The objective of the present study was to analyze the effectiveness associated with first-line targeted therapies, comparing sunitinib with pazopanib. In addition, we estimated the actual cost of medications during the treatment period in the real-life setting.

Most of the 475 patients included in the analysis were treated with sunitinib (81\%) in the first line. A disproportion was expected given that pazopanib was approved in late 2011 by Health Canada (sunitinib had been approved in $2007^{15}$ ). Most patients had an ECOG PS of 0 or $1(85.8 \%)$, which is in line with eligibility criteria for access to targeted therapies in Canadian provinces. The number of patients with metastasis at diagnosis $(52.8 \%)$ was much higher than the numbers published in other studies $(30 \%$ on average) $)^{1,3,4}$.

In the first line, the TTTs for sunitinib and pazopanib before initiation of subsequent treatment were significantly different $(p<0.001)$. That finding could be linked to scheduling adjustments, which were observed mostly in the sunitinib group. In subsequent-line treatment, the TTT varied (3.25-8.04 months), but samples were small, given that only $40 \%$ of the patients received subsequent-line treatment. Several studies looked into the TTT of subsequent-line treatment with inhibitors of mTOR (the mechanistic target of rapamycin), which ranged from 4.9 months to 9.7 months in prospective studies and from 1.4 months to 5.5 months in retrospective studies ${ }^{16-20}$.

A statistically significant difference of 11 months in os was seen between sunitinib and pazopanib in the first line (32 months vs. 21 months respectively). That result differs from the median os observed in the phase III RCT comparing sunitinib with pazopanib (29.1 months vs. 28.3 months respectively) ${ }^{10}$. In previous observational studies, the median os was 17.2-22.3 months in the sunitinib group and 19.6-22.6 months in the pazopanib group ${ }^{21,22}$. The clear difference in survival between the observational studies and the main RCT can be linked to differences in patient characteristics resulting from the more restrictive inclusion and exclusion criteria in clinical trials. For instance, the observational studies included patients with a wider range of disease characteristics, such as brain metastasis or non-clear cell histology, which were not included in the Motzer et al. RCT $^{12}$. However, the median os observed with sunitinib in our analysis was much longer than that reported in other observational studies ${ }^{21,22}$. The factor that can possibly explain the difference is dose scheduling in patients treated with sunitinib. One particular practice in sunitinib dose adjustment has patients starting with, or 
later transitioning to, an alternative schedule of 2 weeks on and 1 week off $(2 / 1)$ or an even more individualized dose than the recommended 4 weeks on and 2 weeks off $(4 / 2)$. Many studies have evaluated the efficacy of the $2 / 1$ schedule compared with the $4 / 2$ schedule and found similar or improved os. In fact, Atkinson et al. ${ }^{23}$ demonstrated a median os of 17.7 months with the traditional schedule and 33.0 months with the alternative schedules $(p<0.0001)$. In another Canadian study, impressive progression-free survival data emerged with the use of a very individualized schedule, and some of those patients would be included in the present study cohort, given that they would also have been part of $\mathrm{CKCis}^{24}$.

Despite the adjusted model, some residual indication bias could still be present. Certain clinical variables, such as those included in the International Metastatic Renal Cell Carcinoma Database Criteria, were captured either not at all or incompletely by our database, rendering it infeasible to account for them. However, Lalani et al. ${ }^{25}$ looked into the Criteria and adjusted the HR of sunitinib compared with pazopanib for those variables, still finding that sunitinib was an independent predictor of survival (adjusted HR: 0.60; 95\% CI: 0.38 to 0.94$)$. Also, several of the International Metastatic Renal Cell Carcinoma Database Criteria were also found to be independently associated with survival, but not confounding factors. Other factors that might account for the os difference between pazopanib and sunitinib in our cKcis database is the trend toward increased age and poorer Ps in the pazopanib group; however, our regression model adjusted for those variables. Some comorbidities such as hypertension and hepatic disease were also not included in our analysis, potentially inducing an indication bias in choice of therapy.

The effect, in clinical practice, of crossing over from one treatment to another, or of using alternative therapies after discontinuation, might explain the difference between our os results and the ones reported in phase III RCTS and observational studies ${ }^{12,21,26-30}$. Many variables, such as time to first-line targeted treatment of less than 1 year, were found to predict mortality. That observation is likely confounded by physician choice to keep patients with a favourable prognosis on observation longer before initiating targeted therapy. Another observational study conducted by Lalani et al. ${ }^{25}$ using the cKcis database compared the clinical efficacy of sunitinib with that of pazopanib. That study differs from ours in that it did not limit the analysis to patients with clear cell histology. In addition, we looked into the effect of various potentially prognostic or confounding variables (such as metastasis location, ECOG PS, age, and time to initiation of treatment) as presented in the Cox regression analysis, and we analyzed the conditional survival of patients beyond 1 year of therapy initiation.

When conditional survival analysis was used to estimate the median os of patients 1 year after treatment initiation, the difference between the two targeted agents was even greater than the difference in os from the initiation of first-line therapy. The choice of therapy was the only factor associated with risk of death when analyzing conditional survival at 1 year. Consequently, it seems that, beyond 12 months of therapy, the predictive value of baseline characteristics for mortality is reduced. However, it is important to note that the sample size had decreased after 1 year, because many patients had died or were lost to follow-up, and therefore statistical significance was not met for some of the covariates. In fact, after 1 year, $73.8 \%$ of patients in the sunitinib group and $72.7 \%$ of those in the pazopanib group were still alive.

The cost of treating patients with targeted therapy is substantial: the median cost of up to 2 lines of therapy was $\$ 56,476$ (IQR: $\$ 23,738-\$ 130,447$ ) for the sunitinib group $-\$ 10,224$ higher than for the pazopanib group. A recent Canadian study had estimated a very similar difference in the cost of treatment with pazopanib and with sunitinib in the first-line setting $(\$ 10,293)^{31}$. It is worth mentioning that the unit prices included in our analysis reflect the drug list of the Province of Quebec, which could differ from prices in other Canadian provinces and do not reflect any product listing agreement that might have been negotiated between the manufacturers and provincial institutions.

The strengths of our analysis include its large multicentre database focused on patients treated with targeted therapies at several Canadian academic hospitals. Given the diverse patient population drawn from various regions and centres, the results should reflect the real-life management of mRcc in the Canadian academic setting.

Some limitations of our study are worth mentioning. One is the imbalance in the proportions of patients treated in the first-line setting with sunitinib and with pazopanib. Our study included only academic centres, which could be seen as selection bias, because treatment patterns and patient characteristics might vary from those seen in the community setting. To allow for comparisons with RCTs, only patients with clear cell histology were included in our study; however, in real life, targeted treatments are known to be used in patients having non-clear cell mRcc, which is not reflected in the present work ${ }^{32,33}$. Furthermore, dose adjustment was not captured in our analysis, because only full-dose therapy was considered. Not accounting for such adjustments could affect the estimated costs because dose reductions are linked with a lower cost for medication. In addition, patients are in some cases treated with subsequent lines of therapy, and their os cannot be attributed solely to their first-line therapy. It is important consider how the overall management of patients directly affects their TTT and os. However, the use of subsequent treatments was proportionally similar in both groups (approximately 42\%) and so the effects of additional treatment were equivalent in the two groups. Also, our study looked only at drug cost and not the cost of other medical care such as management of side effects, hospitalizations, clinic visits, and so forth.

Significant advances such as targeted therapies provide incremental improvement in the lives of patients, but come with high costs and accessibility challenges in Canada. Nevertheless, even with the clinical advances of the past decade, many patients are still not responding to targeted agents. Thus, there is still an unmet medical need in mRCc, mainly because of intrinsic resistance to targeted therapies ${ }^{34,35}$. Finally, real-world evidence can provide guidance by setting benchmarks for drugs under investigation and by setting a foundation for understanding the actual cost related to the use of pharmacotherapy. 


\section{CONCLUSIONS}

Using real-world data, the present analysis confirms the efficacy of pazopanib and sunitinib in the first-line setting, with better os being observed in the sunitinib group. Given that pharmacotherapy and the cost of that therapy are both expanding in mRCc, additional studies covering the whole disease spectrum in the real-life setting should be conducted to optimize management of mrcc.

\section{ACKNOWLEDGMENTS}

This work has previously been presented as meeting abstracts, as follows: Nazha S, Tanguay S, Dragomir A, et al. Cost and management of targeted treatments for metastatic renal cell carcinoma patients in Canada. Presented at the Canadian Urological Association 72nd Annual Meeting; 24-27 June 2017; Toronto, ON; Nazha S, Tanguay S, Dragomir A, et al. Clinical and economic outcomes of drug utilization in clear cell metastatic RCC: a real world evaluation. Presented at the Canadian Center for Applied Research in Cancer Control 2016 Conference; 8-9 May 2016; Toronto, ON; Nazha S, Tanguay S, Dragomir A, et al. Cost and management of targeted treatments for metastatic renal cell carcinoma patients in Canada. Presented at the 19th Annual International Society for Pharmacoeconomics and Outcomes Research European Congress; 29 October-2 November 2016; Vienna, Austria.

\section{CONFLICT OF INTEREST DISCLOSURES}

We have read and understood Current Oncology's policy on disclosing conflicts of interest, and we declare the following interests: MJ has received consulting fees from Pfizer and Ipsen; GB has received consulting fees from Pfizer and Novartis and grants from Pfizer; NB has received consulting fees from Pfizer, Novartis, Bristol-Myers Squibb, Ipsen, and Eisai; EL has received speaker fees from Pfizer and funding from Janssen and Astellas for grants; and DH has received consulting fees from Pfizer and Novartis. The remaining authors have no conflicts of interest to disclose.

\section{AUTHOR AFFILIATIONS}

*McGill University Health Centre, Montreal, QC; ${ }^{\dagger}$ McMaster University, Hamilton, ON; ${ }^{\circ}$ Princess Margaret Cancer Centre, Toronto, ON; ${ }^{\S}$ University of British Columbia, Vancouver, BC; "Dalhousie University and QEII Health Sciences Centre, Halifax, NS; "Sunnybrook Health Sciences Centre, University of Toronto, Toronto, ON; **Tom Baker Cancer Centre, University of Calgary, Calgary, AB; ${ }^{\dagger \dagger}$ Centre hospitalier de l'Université de Montréal, University of Montreal, Montreal, QC; ${ }^{\ddagger \ddagger}$ University of Ottawa, Ottawa, ON; ${ }^{\$}$ Cross Cancer Institute, University of Alberta, Edmonton, $\mathrm{AB}$; $|\|| \mathrm{Centre}$ hospitalier universitaire de Québec, University of Laval, Quebec City, QC.

\section{REFERENCES}

1. Gupta K, Miller JD, Li JZ, Russell MW, Charbonneau C. Epidemiologic and socioeconomic burden of metastatic renal cell carcinoma (mRcc): a literature review. Cancer Treat Rev 2008;34:193-205.

2. Canadian Cancer Society's Advisory Committee on Cancer Statistics. Canadian Cancer Statistics 2015. Toronto, ON: Canadian Cancer Society; 2015.

3. Flanigan RC, Campbell SC, Clark JI, Picken MM. Metastatic renal cell carcinoma. Curr Treat Options Oncol 2003;4:385-90.

4. Lam JS, Leppert JT, Belldegrun AS, Figlin RA. Novel approaches in the therapy of metastatic renal cell carcinoma. World $J$ Urol 2005;23:202-12.

5. Patard JJ, Kim HL, Lam JS, et al. Use of the University of California Los Angeles Integrated Staging System to predict survival in renal cell carcinoma: an international multicenter study. J Clin Oncol 2004;22:3316-22.
6. North SA, Basappa N, Basiuk J, et al. on behalf of the Canadian Kidney Cancer Forum 2015. Management of advanced kidney cancer: Canadian Kidney Cancer Forum consensus update. Can Urol Assoc J 2015; 9:164-70.

7. Escudier B, Porta C, Schmidinger M, et al. on behalf of the ESMo Guidelines Committee. Renal cell carcinoma: ESMO clinical practice guidelines for diagnosis, treatment and follow-up. Ann Oncol 2016;27(suppl 5):v58-68. [Erratum in: Ann Oncol 2017;28(suppl 4):iv167-8]

8. Motzer RJ, Jonasch E, Agarwal N, et al. Kidney cancer, version 3.2015. J Natl Compr Canc Netw 2015;13:151-9.

9. Ljungberg B, Bensalah K, Canfield S, et al. EAU guidelines on renal cell carcinoma: 2014 update. Eur Urol 2015;67:913-24.

10. Motzer RJ, Hutson TE, McCann L, Deen K, Choueiri TK. Overall survival in renal-cell carcinoma with pazopanib versus sunitinib. N Engl J Med 2014;370:1769-70.

11. Motzer RJ, Rini BI, McDermott DF, et al. Nivolumab for metastatic renal cell carcinoma: results of a randomized phase II trial. J Clin Oncol 2015;33:1430-7.

12. Motzer RJ, Hutson TE, Cella D, et al. Pazopanib versus sunitinib in metastatic renal-cell carcinoma. $N$ Engl J Med 2013;369:722-31.

13. Escudier B, Tannir NM, McDermott DF, et al. CheckMate 214: Efficacy and safety of nivolumab + ipilimumab $(\mathrm{N}+\mathrm{I}) \mathrm{v}$ sunitinib (s) for treatment-naïve advanced or metastatic renal cell carcinoma (mRCC), including IMDC risk and PD-L1 expression subgroups [abstract LBA4]. Ann Oncol 2017;28(suppl 5):.

14. Zhang X, Loberiza FR, Klein JP, Zhang MJ. A SAS macro for estimation of direct adjusted survival curves based on a stratified Cox regression model. Comput Methods Programs Biomed 2007;88:95-101.

15. Health Canada, Health Products and Food Branch. Summary Basis of Decision: Votrient. Ottawa, ON: Health Canada; 2010. [Available online at: http://www.hc-sc.gc.ca/dhp-mps/ alt_formats/pdf/prodpharma/sbd-smd/phase1-decision/ drug-med/sbd_smd_2010_votrient_128332-eng.pdf; cited 8 October 2018].

16. Motzer RJ, Escudier B, Oudard S, et al. Phase 3 trial of everolimus for metastatic renal cell carcinoma: final results and analysis of prognostic factors. Cancer 2010;116:4256-65.

17. GerullisH,EckeTH, EimerC,HeuckCJ,Otto T.mTor-inhibition in metastatic renal cell carcinoma. Focus on temsirolimus: a review. Minerva Urol Nefrol 2010;62:411-23.

18. Albiges L, Kube U, Eymard JC, et al. Everolimus for patients with metastatic renal cell carcinoma refractory to anti-vEGF therapy: results of a pooled analysis of non-interventional studies. Eur J Cancer 2015;51:2368-74.

19. Weikert S, Kempkensteffen C, Busch J, et al. Sequential mтоR inhibitor treatment with temsirolimus in metastatic renal cell carcinoma following failure of VEGF receptor tyrosine kinase inhibitors. World J Urol 2013;31:805-9.

20. Feinberg BA, Jolly P, Wang ST, et al. Safety and treatment patterns of angiogenesis inhibitors in patients with metastatic renal cell carcinoma: evidence from US community oncology clinics. Med Oncol 2012;29:786-94.

21. Ruiz-Morales JM, Swierkowski M, Wells JC, et al. Firstline sunitinib versus pazopanib in metastatic renal cell carcinoma: results from the International Metastatic Renal Cell Carcinoma Database Consortium. Eur J Cancer 2016;65:102-8.

22. Pal SK, Ghate SR, Li N, et al. Real-world survival outcomes and prognostic factors among patients receiving first targeted therapy for advanced renal cell carcinoma: a sEER-Medicare database analysis. Clin Genitourin Cancer 2017;15:e573-82.

23. Atkinson BJ, Kalra S, Wang X, et al. Clinical outcomes for patients with metastatic renal cell carcinoma treated with alternative sunitinib schedules. J Urol 2014;191:611-18. 
24. Bjarnason GA, Khalil B, Hudson JM, et al. Outcomes in patients with metastatic renal cell cancer treated with individualized sunitinib therapy: correlation with dynamic microbubble ultrasound data and review of the literature. Urol Oncol 2014;32:480-7.

25. Lalani AA, Li H, Heng DYC, et al. First-line sunitinib or pazopanib in metastatic renal cell carcinoma: the Canadian experience. Can Urol Assoc J 2017;11:112-17.

26. Motzer RJ, Hutson TE, Tomczak P, et al. Overall survival and updated results for sunitinib compared with interferon alfa in patients with metastatic renal cell carcinoma.JClin Oncol 2009;27:3584-90.

27. Sternberg CN, Hawkins RE, Wagstaff J, et al. A randomised, double-blind phase III study of pazopanib in patients with advanced and/or metastatic renal cell carcinoma: final overall survival results and safety update. Eur J Cancer 2013;49:1287-96.

28. Matrana MR, Duran C, Shetty A, et al. Outcomes of patients with metastatic clear-cell renal cell carcinoma treated with pazopanib after disease progression with other targeted therapies. Eur J Cancer 2013;49:3169-75.

29. Jonasch E, Signorovitch JE, Lin PL, et al. Treatment patterns in metastatic renal cell carcinoma: a retrospective review of medical records from US community oncology practices. Curr Med Res Opin 2014;30:2041-50.
30. Vogelzang NJ, Hackshaw MD, Hutson TE, et al. First-line and sequential use of pazopanib followed by mammalian target of rapamycin inhibitor therapy among patients with advanced renal cell carcinoma in a US community oncology setting. Clin Genitourin Cancer 2015;13:210-17.

31. Amdahl J, Diaz J, Park J, Nakhaipour HR, Delea TE. Costeffectiveness of pazopanib compared with sunitinib in metastatic renal cell carcinoma in Canada. Curr Oncol2016; 23:e340-54

32. Gore ME, Szczylik C, Porta C, et al. Safety and efficacy of sunitinib for metastatic renal-cell carcinoma: an expandedaccess trial. Lancet Oncol 2009;10:757-63.

33. Kroeger N, Xie W, Lee JL, et al. Metastatic non-clear cell renal cell carcinoma treated with targeted therapy agents: characterization of survival outcome and application of the International mRCC Database Consortium criteria. Cancer 2013;119:2999-3006.

34. Busch J, Seidel C, Kempkensteffen C, et al. Sequence therapy in patients with metastatic renal cell carcinoma: comparison of common targeted treatment options following failure of receptor tyrosine kinase inhibitors. Eur Urol 2011;60:1163-70.

35. Heng DY, Mackenzie MJ, Vaishampayan UN, et al. Primary anti-vascular endothelial growth factor (VEGF)-refractory metastatic renal cell carcinoma: clinical characteristics, risk factors, and subsequent therapy. Ann Oncol 2012;23:1549-55. 